5. J. L. Kelley, Banach spaces with the extension property, Trans. Amer. Math. Soc. 72 (1952), 323-326.

6. L. Nachbin, $A$ theorem of the Hahn-Banach type for linear transformations, Trans. Amer. Math. Soc. 68 (1950), 28-46.

7. - Some problems in extending and lifting continuous linear transformations, Proc. Internat. Sympos. Linear Spaces (Jerusalem, 1960), pp. 340-351, Jerusalem Academic Press, Jerusalem, 1961.

8. A. Pełczyński and Z. Semadeni, Spaces of continuous functions. III, Studia Math. 8 (1959), 211-222.

UNiversity of CALIForNia, BERKELEy

\title{
GEODESICS AND LEBESGUE AREA
}

\section{EDWARD SILVERMAN ${ }^{1}$}

A method of stretching a Fréchet surface by various amounts, without increasing its Lebesgue area, was introduced in [S2]. The stretched surfaces derived from a given continuous surface are all continuous and, in general, there is no largest such surface. In this paper we admit discontinuous surfaces and this enables us to stretch a surface to its maximum extension. If the maximum extension is continuous, then its (Lebesgue) area is the same as the area of the surface being stretched. In general, if the area of the maximum extension is defined to be the supremum of all continuous surfaces which are not bigger, in the sense of Kolmogoroff's Principle applied to surfaces, then this area of the maximum extension of a given continuous surface agrees with the Lebesgue area of the surface. Furthermore, if two continuous surfaces are so related that the pseudo-geodetic distances between corresponding pairs of points are equal, then the maximum stretched surfaces derived from these two are isometric, which implies that the two original surfaces have the same Lebesgue area. (The pseudo-geodetic distance agrees with geodetic distance for light maps.) An example of R. D. Anderson, modified by R. F. Williams, gives a monotone map of a square onto another square with the property that the geodetic distance between every distinct pair of points is infinite. Thus, it is neither desirable nor possible for the preceding result to hold for geodetic as well as for pseudo-geodetic distance.

Presented to the Society, August 30, 1962; received by the editors April 25, 1963.

1 This research was supported in part by National Science Foundation Grant No. GP634. 
The definition of Lebesgue area to be used is given for surfaces in $m$, the space of bounded sequences [B], and isometric surfaces have the same area. Since each separable metric space is isometric with a subset of $m$, we can define Lebesgue area for surfaces in metric spaces. If the metric space happens to be Euclidean space then this area agrees with the usual Lebesgue area on each surface for which that area agrees with the Geocze area. These areas are known to agree for all 2-dimensional surfaces and for a wide class of $n$-dimensional surfaces, $n>2[\mathrm{C}],[\mathrm{F}]$.

Let $a_{1}, \cdots, a_{n} \in m$, let $R$ be the space and $P$ the parallelepiped determined by these vectors. If volume is to behave properly with respect to affine transformations, then the volume of $P$ will be determined if the volume of any nondegenerate parallelepiped in $R$ is prescribed. It is not difficult to see that there exists a parallelepiped $K$ containing the unit sphere in $R$ whose volume does not exceed that of any other such figure. We set volume $K=2^{n}$. Alternatively, the volume of $P$ is given by sup $\operatorname{det}_{i_{1}}, \cdots, i_{n}\left(a_{i}^{i_{j}}\right)$. If $E$ is Euclidean $n$ space then there exists a linear transformation $U: E \rightarrow m$ that is an isometry [S1]. It is evident, now, that volume $U(\Delta)=$ volume $\Delta$ whenever $\Delta$ is an $n$-simplex in $E$.

Let $Q$ be an $n$-cell and let $C$ be the space of continuous functions on $Q$ into $m$. If $z \in C$ and if $Q$ can be subdivided into a finite number of $n$-simplexes on each of which $z$ is linear then $z$ is quasilinear and the elementary area of $z, \varepsilon z$, is the sum of the volumes of the images, under $z$, of the $n$-simplexes of linearity of $z$.

If $x \in C$ then $L x$, the Lebesgue area of $x$, is given by $L x$ $=\lim _{\inf _{z \rightarrow x}} \varepsilon z$, where $z$ is quasilinear and convergence is in $C$.

If $x \in C$ let $x^{\prime}$ be defined on $Q \times Q$ by

$$
x^{\prime}(p, q)=\|x(p)-x(q)\| \quad \text { for all } p, q \in Q .
$$

We shall make use of the following properties of Lebesgue area, the proofs of which differ only in detail from the 2-dimensional case given in $[\mathbf{S 1}]$ :

(1) $L x \leqq L y$ if $x^{\prime} \leqq y^{\prime}$ (Kolmogoroff's Principle).

(2) $L x=\sum_{i=1}^{k} L\left(x \mid \Delta_{i}\right)$ if $x$ is Lipschitzian and if $Q$ is subdivided in the $n$-simplexes $\Delta_{1}, \cdots, \Delta_{k}$.

(3) $L$ is lower semi-continuous on $C$.

(4) If $x \in C$ then there exists a sequence $\left\{z^{p}\right\}$ of quasilinear functions such that $z^{p} \rightarrow x$ and $\varepsilon z^{p} \rightarrow L x$.

Let $S$ be the collection of functions $X$ on $Q \times Q$ into $[0, \infty]$ satisfying $X(p, p)=0, X(p, q)=X(q, p)$ and $X(p, q)+X(q, r) \geqq X(p, r)$ for all $p, q, r \in Q$. Let $S_{e}=\{X \in S \mid X$ is continuous $\}$. Evidently $x^{\prime} \in S_{c}$ whenever $x \in C$. 
Let $\left\{p_{i}\right\}$ be a fixed sequence, dense in $Q$. If $X \in S_{c}$ let $X^{*}(p)$ $=\left\{X\left(p_{i}, p\right)\right\}$ for all $p \in Q$. Then $X^{*} \in C$ and $\left(X^{*}\right)^{\prime}=X$, [S2].

If $f$ is continuous on a closed interval into $m$ and if $k$ is a positive integer, then let $\mu_{k} f$ be the maximum length of any $k$-gon inscribed in $f$. Then $\mu_{k} f^{p} \rightarrow \mu_{k} f$ if $f^{p}$ converges to $f$ in the sense of Fréchet [S2].

Let $H(p, q)$ be the set of functions $f$ continuous on $[0,1]$ into $Q$ with $f(0)=p$ and $f(1)=q$.

If $x \in C$ then $x_{k} \in C$ is defined as follows: Let

$$
M_{k}(p, q)=\inf \left\{\mu_{k} x f \mid f \in H(p, q)\right\} .
$$

Then $M_{k} \in S_{c}$ and so $x_{k}=M_{k}^{*} \in C$, [S2]. It is immediate that $x^{\prime} \leqq x_{k}^{\prime}=M_{k} \leqq x_{k+1}^{\prime}$ and that $\left\|x_{k}-y_{k}\right\| \leqq 2 k\|x-y\|$. Furthermore, $x_{k}$ is Lipschitzian if $x$ is.

LEMMA 1. $L x_{k}=L x$.

The proof of this lemma for 2-dimensional Lebesgue area and any $\nu$-length appears in [S2]. The same proof holds for $n$-dimensional area. That the lemma holds for $x$ quasilinear follows from (2) and (1). Now let $x \in C$ and $\left\{z^{p}\right\}$ be as in (4). We have $\left\|z_{k}^{p}-x_{k}\right\| \leqq 2 k\left\|z^{p}-x\right\|$. Thus, by (3), what we have already proved, and by (1), $L x_{k}$ $\leqq \lim \inf L z_{k}^{p}=\lim \inf \varepsilon z^{p}=L x \leqq L x_{k}$.

If $E \subset m, E^{1} \subset m, k>0$ and $\tau$ is a function on $E$ to $E^{1}$ such that $\|\tau p-\tau q\| \leqq k\|p-q\|$ for all $p, q \in E$, then $\tau$ can be extended to all of $m$ as a Lipschitz map with constant $k,[\mathrm{~S} 1]$.

LEMma 2. If $x, y \in C$ then there exists $w \in C$ such that $\|w-x\| \leqq d$ and $w^{\prime} \leqq y^{\prime}$ where

$$
d=\sup \left\{x^{\prime}(p, q)-y^{\prime}(p, q) \mid p, q \in Q\right\} .
$$

Proof. Let $V(p, q)=\min \left\{x^{\prime}(p, r)+y^{\prime}(r, q) \mid r \in Q\right\}$. The triangle inequality gives $x^{\prime}-d \leqq V \leqq x^{\prime}$ and $|V(p, q)-V(p, t)| \leqq y^{\prime}(q, t)$. Let $v(p)=\left\{V\left(p_{i}, p\right)\right\}$ and $z=x^{\prime *}$. Since $v^{\prime} \leqq y^{\prime}$ and $z^{\prime}=x^{\prime}$, both $v$ and $z$ are in $C$. Furthermore, the isometry $\tau$ from range $z$ onto range $x$, $\tau(z(p))=x(p)$ for all $p \in Q$, can be extended to a contraction $T$ of $m$. Let $w=T v$. Then $\|w-x\| \leqq\|v-z\|=\sup _{p} \sup _{i}\left|V\left(p_{i}, p\right)-x^{\prime}\left(p_{i}, p\right)\right|$ $=\sup _{p} \sup _{i}\left[x^{\prime}\left(p_{i}, p\right)-V\left(p_{i}, p\right)\right] \leqq d$ and $w^{\prime} \leqq v^{\prime} \leqq y^{\prime}$.

If $g$ is a real-valued function then let $g^{+}$be that function which agrees with $g$ where $g$ is non-negative and vanishes where $g$ is nonpositive.

Theorem 1. If $x \in C$ then $L x \leqq \lim \inf _{\left(x^{\prime}-z^{\prime}\right)^{+} \rightarrow 0}$ Lz where $z \in C$ and the convergence is uniform. 
Proof. Let $M<L x$. Then there exists $\delta>0$ such that $L w>M$ whenever $w \in C$ and $\|w-x\| \leqq \delta$. Now suppose that $\left(x^{\prime}-z^{\prime}\right)^{+} \leqq \delta$. Then, by the last lemma, there exists $w \in C$ with $\|w-x\| \leqq \delta$ and $w^{\prime} \leqq z^{\prime}$. Hence, $L z \geqq L w>M$.

It is more convenient to define an area on $S$ than to extend $m$ so as to permit a surface to have infinite diameter. Let $L^{\prime}$ be defined on $S$ by $L^{\prime} X=\sup \left\{L z \mid z \in C\right.$ and $\left.z^{\prime} \leqq X\right\}$.

We introduce the following notation: If $x, y \in C$ then $X=\lim x_{k}^{\prime}$, $X_{1}=\lim \left(x_{1}\right)_{k}^{\prime}$ and $Y=\lim y_{k}^{\prime}$. The capital letters will only be used in this way. Thus, they are used only if the corresponding small letters represent elements in $C$.

Theorem 2. If $x \in C$ then $L^{\prime} X=L x$.

Proof. That $L^{\prime} X \geqq L x$ follows from the definitions. Let $z \in C$ and $\boldsymbol{z}^{\prime} \leqq X$. By a theorem of Dini, $x_{k}^{\prime} \wedge \boldsymbol{z}^{\prime} \rightarrow \boldsymbol{z}^{\prime}$, where the convergence is uniform. Hence, $\left(z^{\prime}-x_{k}^{\prime}\right)^{+} \rightarrow 0$ and $L z \leqq \lim$ inf $L x_{k}=L x$. Thus, $L^{\prime} X \leqq L x$.

Corollary. If $X=Y$ then $L x=L y$.

The purpose of the rest of the paper is to show that $X(p, q)$ $=X_{o}(p, q)$, the pseudo-geodetic distance between $p$ and $q$ under $x$.

LEMмa 3. $X(p, q)=0$ if and only if $x_{1}^{\prime}(p, q)=0$.

Proof. Let $\theta(p, q)=\inf \operatorname{diam} x(\gamma)$, where $\gamma$ is a continuum in $Q$ containing $p$ and $q$. By a theorem of Zoretti, $\theta(p, q)=0$ implies that $p$ and $q$ belong to the same continuum of constancy of $x$ [C]. Now let $x_{1}^{\prime}(p, q)=0$. Since $\theta \leqq x_{1}^{\prime}$, there is a continuum $\gamma \subset Q$ containing $p$ and $q$ on which $x$ is constant. If $\eta>0$ is chosen so that $\|r-s\|<\eta$ implies $x^{\prime}(r, s)<\epsilon /(2 k)$ and if $g \in H(p, q)$ with range $g$ contained in an $\eta$ neighborhood of $\gamma$ then $\mu_{k} x \gamma<\epsilon$. Hence, $x_{k}^{\prime}(p, q)=0$ for all $k$ and $X(p, q)=0$. If $X(p, q)=0$ then $x_{1}^{\prime}(p, q)=0$ since $0 \leqq x_{1}^{\prime} \leqq X$.

The proof of the lemma shows that $x_{1}$ is the monotone factor in a monotone-light factorization of $x$.

LEMMA $4 .\left(x_{1}\right)_{k}^{\prime} \leqq x_{2 k}^{\prime}$.

Proof. Let $p, q \in Q$ and $f \in H(p, q)$. There exist $0 \leqq t_{1} \leqq \cdots$ $\leqq t_{k+1} \leqq 1$ such that $\mu_{k} x_{1} f=\sum_{i=1}^{k}\left\|x_{1} f\left(t_{i+1}\right)-x_{1} f\left(t_{i}\right)\right\|$. Now there exist $u_{j}, j=1, \cdots, 2 k, t_{i} \leqq u_{2 i-1} \leqq u_{2 i} \leqq t_{i+1}$, such that $\left\|x_{1} f\left(t_{i+1}\right)-x_{1} f\left(t_{i}\right)\right\|$ $\leqq\left\|x f\left(u_{2 i}\right)-x f\left(u_{2 i-1}\right)\right\|$. Hence, $\mu_{k} x_{1} f \leqq \mu_{2 k} x f$ and the lemma follows.

Lemma 5. $X_{1}=X$. 
Proof. That $X_{1} \geqq X$ is evident. On the other hand $X_{1}=\lim \left(x_{1}\right)_{k}^{\prime}$ $\leqq \lim x_{2 \mathbf{k}}^{\prime}=X$.

If $x \in C$ and if $p, q \in Q$ then let $G(x, p, q)$ be the set of all continuous functions $\gamma$ on $[0,1]$ into range $x_{1}$ such that $\gamma(0)=x_{1}(p)$ and $\gamma(1)$ $=x_{1}(q)$.

If $x=\xi \eta$ is a monotone-light factorization of $x$ and if $p, q \in Q$, then let $X_{o}(p, q)=\inf \{$ length $\xi \gamma \mid \gamma$ is continuous on $[0,1]$ into range $\eta$, $\gamma(0)=\eta(p)$ and $\gamma(1)=\eta(q)\}$. Let $\lambda$ be the light map defined by $x=\lambda x_{1}$. Evidently $X_{g}(p, q)=$ inf $\{$ length $\lambda \gamma \mid \gamma \in G(x, p, q)\}$. We shall always use the monotone-light factorizations $x=\lambda x_{1}$ and $x_{1}=i x_{1}$, where $i$ is the identity on range $x_{1}$. We shall write $X_{1 。}$ for $Y_{\vartheta}$ if $y=x_{1}$.

Lemma 6. Let $\gamma \in G(x, p, q)$. Then $\left\|x_{1}(p)-x_{1}(q)\right\| \leqq \operatorname{diam} \lambda \gamma$.

Proof. Let $\epsilon>0$. There exists $\delta>0$ such that $x^{\prime}(r, s)<\epsilon / 2$ whenever $\|r-s\|<\delta$. Let $f \in H(p, q)$ such that range $f$ is contained in a $\delta$ neighborhood of $\Gamma=\left\{r \in Q \mid x_{1}(r) \in\right.$ range $\left.\gamma\right\}$. There exist $0 \leqq u_{1} \leqq u_{2} \leqq 1$ such that diam $x f=\left\|x f\left(u_{2}\right)-x f\left(u_{1}\right)\right\|$ and there exist $p_{1}, p_{2} \in \Gamma$ such that $\left\|f\left(u_{1}\right)-p_{1}\right\|<\delta$ and $\left\|f\left(u_{2}\right)-p_{2}\right\|<\delta$. Hence, $\left\|x_{1}(p)-x_{1}(q)\right\|$ $\leqq\left\|x f\left(u_{2}\right)-x f\left(u_{1}\right)\right\| \leqq\left\|x\left(p_{1}\right)-x\left(p_{2}\right)\right\|+\epsilon=\left\|\lambda x_{1}\left(p_{1}\right)-\lambda x_{1}\left(p_{2}\right)\right\|+\epsilon$ $\leqq \operatorname{diam} \lambda \gamma+\epsilon$.

Corollary 1. length $\lambda \gamma=$ length $\gamma$.

Corollary 2. $X_{1,}=X_{g}$.

LEMMA 7. If $D$ is a compact metric space, if $f_{k}$ is continuous on a closed interval into $D$ and if there exists $M$ such that $\mu_{k} f_{k} \leqq M$ for each positive integer $k$, then a subsequence of $\left\{f_{k}\right\}$ converges in the sense of Fréchet. [S2].

This lemma can be proved directly or from a theorem of Fréchet

Lemma 8. If $f_{k}$ converges to $f$ in the sense of Fréchet then length $f$ $\leqq \lim \inf \mu_{k} f_{k}$.

Proof. Let $M<$ length $f$. There exists $k_{0}$ such that $\mu_{k_{0}} f>M$ and there exists $k_{1}>k_{0}$ such that $\mu_{k_{0}} f_{k}>M$ whenever $k>k_{1}$. Thus, if $k>k_{1}, \mu_{k} f_{k} \geqq \mu_{k_{0}} f_{k}>M$ and so $\lim \inf \mu_{k} f_{k} \geqq$ length $f$.

Theorem 3. If $p, q \in Q$ then $X(p, q)=\min \{$ length $\gamma \mid \gamma \in G(x, p, q)\}$ $=X_{g}(p, q)$ where the minimum is infinite if length $\gamma=\infty$ for each $\gamma \in G(x, p, q)$.

Proof. Let us first suppose that $X(p, q)$ is finite. Then there exists $f_{k} \in H(p, q)$ such that $\mu_{k} x_{1} f_{k}<\left(x_{1}\right)_{k}^{\prime}(p, q)+1 / k \leqq X(p, q)+1 / k$ for all $k$. By Lemma 7 there exists $\gamma_{0}$, easily seen to be in $G(x, p, q)$, to which 
a subsequence of $\left\{x_{1} f_{k}\right\}$ converges in the sense of Fréchet. By Lemma 8 , length $\gamma_{0} \leqq X(p, q)$. Hence, $X_{g}(p, q) \leqq X(p, q)$ whether or not $X(p, q)$ is finite and there is no loss in generality in supposing that $X_{o}(p, q)<\infty$. Let $\epsilon>0$ and $\gamma$ be a rectifiable element of $G(x, p, q)$. There exists $\delta>0$ such that the oscillation of $\gamma$ over any interval, of length less than $\delta$, is less than $\epsilon$. Let $k$ be so large that $k \delta>1$ and let $t_{i}=i / k, i=0,1, \cdots, k$. There exists $\eta>0$ such that $x^{\prime}(r, s)<\epsilon$ whenever $\|r-s\|<\eta$. Let $\Gamma_{i}=\left\{r \in Q \mid x_{1}(r) \in \gamma\left(\left[t_{i-1}, t_{i}\right]\right)\right\}$, let $q_{0}=p, q_{k}=q$ and let $q_{i}$ be chosen so that $x_{1}\left(q_{i}\right)=\gamma\left(t_{i}\right)$ for $i=1, \cdots, k-1$. Let $f_{i}$ be quasilinear on $\left[t_{i-1}, t_{i}\right]$ into an $\eta$-neighborhood of $\Gamma_{i}$ such that $f_{i}\left(t_{i-1}\right)$ $=q_{i-1}$ and $f_{i}\left(t_{i}\right)=q_{i}$. Now define $f \in H(p, q)$ by $f \mid\left[t_{i-1}, t_{i}\right]=f_{i}$. It $0 \leqq u_{1} \leqq \cdots \leqq u_{k+1} \leqq 1$ then let $v_{i}=t_{j}$, where $t_{j} \leqq u_{i}<t_{j+1}$. Now

$$
\begin{aligned}
& \sum\left\|x f\left(u_{i+1}\right)-x f\left(u_{i}\right)\right\| \\
& \quad \leqq \sum\left[\left\|x f\left(u_{i+1}\right)-x f\left(v_{i+1}\right)\right\|+\left\|x f\left(v_{i+1}\right)-x f\left(v_{i}\right)\right\|+\left\|x f\left(v_{i}\right)-x f\left(u_{i}\right)\right\|\right] \\
& \quad<2 k \epsilon+\text { length } \gamma .
\end{aligned}
$$

Thus, $x_{k}^{\prime}(p, q) \leqq$ length $\gamma$ for all $k$ and so $X(p, q) \leqq X_{g}(p, q)$. Finally, length $\gamma_{0} \leqq X(p, q) \leqq X_{o}(p, q) \leqq$ length $\gamma_{0}$.

\section{REFERENCES}

B. S. Banach, Théorie des opérations linéaires, Monografje Matematyczne, Warsaw, 1932 .

C. L. Cesari, Surface area, Annals of Mathematics Studies No. 35, Princeton Univ. Press, Princeton, N. J., 1956.

F. H. Federer, Currents and area, Trans. Amer. Math. Soc. 98 (1961), 204-233.

S1. E. Silverman, Definitions of Lebesgue area for surfaces in metric spaces, Riv. Mat. Univ. Parma 2 (1951), 47-76.

S2. — An intrinsic property of Lebesgue area, Riv. Mat. Univ. Parma 2 (1951), 195-201.

Purdue University 